\title{
Lymphatic Filariasis: An Immunologic Perspective
}
Authors:
*Joshua Mandanas
University of Perpetual Help, Dr. Jose G. Tamayo Medical University Santo Niňo, Biňan, Laguna, Philippines
${ }^{*}$ Correspondence to mandanas.joshua@uphsl.edu.ph
Disclosure: $\quad$ The author has declared no conflicts of interest.
Acknowledgements: The author would like to acknowledge University of Perpetual Help - Dr. Jose G. Tamayo Medical University and University of the Philippines Manila.
Received:
31.10 .20
Accepted:
10.05.21
Keywords:
Brugia malayi, helminth, immunology, lymphatic filariasis, Wuchereria bancrofti.
Citation:
EMJ Allergy Immunol. 2021;6[1]:71-78.

\section{Abstract}

Introduction: This paper provides an overview of the current immunologic research findings of lymphatic filarial worms, which affect millions of people worldwide.

Objectives: This paper aims to discuss the immunologic features of lymphatic filarial worms. It also aims to highlight their potential anti-inflammatory actions and the use of anti-filarial drugs against COVID-19.

Methods: A literature review was performed to obtain insights on the immunologic features of lymphatic filarial worms.

Results: The CD4+ (Th2) response profile is the main defence against filarial worms. Modulation of human immune responses are primarily mediated by parasite-secreted peptides. Lymphatic filarial worms have anti-inflammatory potentials. Drug repurposing of diethylcarbamazepine, doxycycline, and ivermectin can be looked upon against COVID-19.

Conclusion: Lymphatic filarial worms have several immunologic effects on host immune systems, which promote chronic infection and curtail anti-inflammatory responses. Insights in this paper can serve as a guide for the understanding of immunologic aspects of lymphatic filarial worms.

\section{BASIC EPIDEMIOLOGY}

According to the World Health Organization (WHO), approximately 120 million people situated in the tropical and subtropical regions were affected by lymphatic filariasis.' Walker et al. ${ }^{2}$ noted that the majority of Iymphatic filariasis cases worldwide are caused by Wuchereria bancrofti, while Brugia malayi and Brugia timori cause many of the cases in Asia. The Philippines

has 45 provinces that are endemic for lymphatic filarial worms $W$. bancrofti and B. malayi.

Walker et al. $^{2}$ noted that the migration of people from rural to urban areas contributed to an increase in distribution of $W$. bancrofti and B. malayi. ${ }^{2}$ This leads to an increase in vector breeding sites. Turkington et al. ${ }^{4}$ noted that males are usually affected due to frequent exposure to areas with mosquitos, such as agricultural areas. Specifically, older males (>35 years of age), 
farmers, and those living near rivers have a higher risk of infection. 5,6

\section{BIOLOGY OF THE FILARIAL WORMS}

Filarial nematodes belong to the phylum Nematoda, class Secernentea, order Rhabditida, superfamily Filarioidea, and family Onchocercidae. ${ }^{7}$ They are usually differentiated by the location, habitat of the worms, geographical distribution, periodicity, or time of diagnosis of the microfilaria (offspring) of the adult worms, morphology (presence of sheath, appearance of tail and head regions), plus the type of vectors and periodic biting preferences. Filarial nematodes, in terms of adult worm locations, are usually found in the lymphatics, subcutaneous, and bodily cavities. Table 1 summarises the filarial worms, their microfilarial morphology with periodicity, along with vector details. ${ }^{7-10}$

Filarial nematodes are dioecious and exhibit sexual dimorphism.11,12 Adult males have corked tails with two spicules and are smaller than females. ${ }^{7}$ In the Philippines, Aedes poicilus is the main vector for $W$. bancrofti in most provinces. ${ }^{3}$

Table 1: Different classifications of the filarial nematodes.

\begin{tabular}{|c|c|c|c|c|}
\hline Filarial worm & $\begin{array}{l}\text { Microfilarial } \\
\text { morphology }^{7}\end{array}$ & $\begin{array}{l}\text { Microfilarial } \\
\text { periodicity }^{8}\end{array}$ & Vectors $^{9}$ & $\begin{array}{l}\text { Vector biting } \\
\text { periodicity }\end{array}$ \\
\hline $\begin{array}{l}\text { Lymphatic filarial } \\
\text { worms }\end{array}$ & & & Mosquitos & \\
\hline Wuchereria bancrofti & $\begin{array}{l}\text { Sheath is present; no } \\
\text { nuclei to tail tip }\end{array}$ & $\begin{array}{l}\text { Nocturnal } \\
\text { (10 p.m.-2 a.m.) }\end{array}$ & $\begin{array}{l}\text { Aedes, Anopheles, } \\
\text { and Culex }\end{array}$ & Mostly nocturnal \\
\hline \multirow[t]{2}{*}{ Brugia malayi } & \multirow[t]{2}{*}{$\begin{array}{l}\text { Sheath is present; two } \\
\text { nuclei in tail }\end{array}$} & $\begin{array}{l}\text { Nocturnal } \\
\text { (10 p.m.-2 a.m.) }\end{array}$ & $\begin{array}{l}\text { Aedes, Anopheles, } \\
\text { and Mansonia }\end{array}$ & $\begin{array}{l}\text { Mostly nocturnal; } \\
\text { Mansonia can be sub- } \\
\text { periodic }\end{array}$ \\
\hline & & Sub-periodic & Coquillettidia & Mostly nocturnal \\
\hline Brugia timori & $\begin{array}{l}\text { Sheath is present } \\
\text { (does not stain with } \\
\text { Giemsa); two nuclei at } \\
\text { tip; cephalic space } 3: 1\end{array}$ & $\begin{array}{l}\text { Nocturnal } \\
\text { (10 p.m.-2 a.m.) }\end{array}$ & Anopheles & Mostly nocturnal \\
\hline $\begin{array}{l}\text { Subcutaneous filarial } \\
\text { worms }\end{array}$ & & & Flies and midges & \\
\hline Loa loa & $\begin{array}{l}\text { Sheath is present; } \\
\text { continuous nuclei to } \\
\text { tail tip }\end{array}$ & Diurnal & $\begin{array}{l}\text { Chrysops, fruit fly, } \\
\text { mango fly }\end{array}$ & $\begin{array}{l}9 \text { a.m.-11 a.m. } \\
2 \text { p.m.-4 p.m. }\end{array}$ \\
\hline Onchocerca volvulus & $\begin{array}{l}\text { Unsheathed; no nuclei } \\
\text { at tail tip }\end{array}$ & None & $\begin{array}{l}\text { Simulium or black fly, } \\
\text { buffalo gnat }\end{array}$ & $\begin{array}{l}10 \text { a.m. }-11 \text { a.m. } \\
4 \text { p.m.-6 p.m. }\end{array}$ \\
\hline $\begin{array}{l}\text { Mansonella } \\
\text { streptocerca }\end{array}$ & $\begin{array}{l}\text { Unsheathed; nuclei to } \\
\text { tip of tail and is bent } \\
\text { ('shepherd's crook' } \\
\text { appearance) }\end{array}$ & None & Culicoides (midge) & Dawn and dusk \\
\hline $\begin{array}{l}\text { Mesenteric/Deep } \\
\text { tissues/Skin }\end{array}$ & & & Flies and midges & \\
\hline Mansonella perstans & $\begin{array}{l}\text { Unsheathed; nuclei to } \\
\text { tip of tail }\end{array}$ & None & Culicoides (midge) & Dawn and dusk \\
\hline Mansonella ozzardi & $\begin{array}{l}\text { Unsheathed; no nuclei; } \\
\text { tail is shorter/slender/ } \\
\text { tapered }\end{array}$ & None & $\begin{array}{l}\text { Culicoides (midge), } \\
\text { Simulium }\end{array}$ & Dawn and dusk \\
\hline
\end{tabular}


Other vectors include Anopheles minimus var. flavirostris and Culex spp. Mansonia bonnae and Mansonia uniformis are the main mosquito vectors for B. malayi, although Aedes spp. and Anopheles spp. can be utilised. ${ }^{13}$ Partono et al. ${ }^{14}$ mentioned that Anopheles barbirostris is the vector for $B$. timori. In humans, the third stage filariform enter the skin from mosquito bites and migrate into lymphatic vessels, where they molt into their adult forms for $>1$ month in the case of W. bancrofti and 2-3 weeks for B. malayi. The adults are viviparous in nature (parasite species that lay larva instead of eggs) and release first-stage larva called microfilaria. A gravid (pregnant) female is able to discharge about 50,000 microfilaria per day. ${ }^{7}$ The incubation period is about 8-16 months, with females capable of laying eggs for 5 years, and adults can generally live for 9 years. ${ }^{15,16}$

\section{IMMUNOLOGIC FEATURES OF THE LYMPHATIC FILARIAL WORMS}

\section{Normal Host Response Against Lymphatic Filarial Worms}

The immune response of the human host to filarial infections is characterised by CD4+ (Th2) response, humoral response (antibodies: IgG1, $\operatorname{lgG} 4$, IgM, and IgE), and cytokine response (IL-4, IL-5, IL-9, IL-10, IL-13). Cellular responses involve the action of mast cells, basophils, eosinophils, and macrophages. ${ }^{17}$ Chronic infections involve the action of $T$ regulatory cells and macrophages. Th2 is deemed generally protective for filarial infections.

Macrophage presentation with CD4+ cells during filarial infections activates the latter to induce secretion of cytokines (IL-3, IL-4, IL-9) to activate mast cells, as well as IL-5 for eosinophils and IL-4 to induce plasma cell secretion of antibodies IgM (acute), IgG (chronic), and IgE.

IgG antibodies (IgG1, IgG4, and $\lg M$ ), with their Fab portions, can bind to the surface antigens of filarial worms. The effector cells such as macrophages and eosinophils utilise an antibody-dependent cytotoxicity for destroying parasitic membranes, either by production of nitric oxide, secretion of perforins, or other Iytic enzymes. ${ }^{18}$ The immunological function of IgE entails mast cell degranulation to release eosinophil and neutrophil chemotactic factors for continued parasitic clearance. High levels of IgG4 are indicative of $W$. bancrofti infection while IgG1 can be protective against $B$. malayi.19,20 Platelet activating factors are also released by eosinophils and neutrophils that in turn activate formation of a clot that may block filarial worm migration. ${ }^{21}$

Complement proteins are involved in parasitic membrane lysis, production of opsonins for enhancement of phagocytosis, and anaphylatoxins, which enable mast cells to be activated. Senbagavalli et al. ${ }^{22}$ noted that classical, mannose-binding lectin (MBL), and alternative pathways are activated in filarial infections, with classical and MBL pathways being elevated during evident or active infection. ${ }^{22}$

\section{Immunomodulatory Effects of the Lymphatic Filarial Worms}

Figure 1 summarises the immunobiological features of lymphatic filarial worms. Filarial worms have characteristic secreted products that can affect the immune functioning of the host. Proteins found in filarial worms such as phosphorylcholine (ES-62) can prevent proliferation of CD4+ T cells and B cells..$^{23}$ In this way, the release of activating cytokines such as IL-4 may be hampered, and normal activation of the anti-parasitic mechanisms might not take place. Antibody-dependent cytotoxicity will also be affected. It is also noted that filarial infections, in terms of chronic states, affect the antigen presentation of the human host. Semnani et al. ${ }^{24}$ noted that filarial worms can down-regulate $M H C$ / and // genes.

Analysis of the filarial genome revealed that filarial worms secrete homologues that impair cytokines such as IL-5, IL-16 as well as IFN regulatory factors, and suppress cytokine signalling. ${ }^{23,25}$ Antigen processing, presentation, and immune activation are also affected, with inhibition of proteins and signalling compounds such as serpins, cystatins, indoleamine 2,3-dioxygenase genes, and Wnt signalling regulators. Liu et al. ${ }^{21}$ noted that eicosanoids released from filarial worms can inhibit platelet aggregation.

\section{Immunology of Asymptomatic Infections}

The prelude to a chronic, debilitating, disfiguring lymphatic filariasis is the asymptomatic state. 


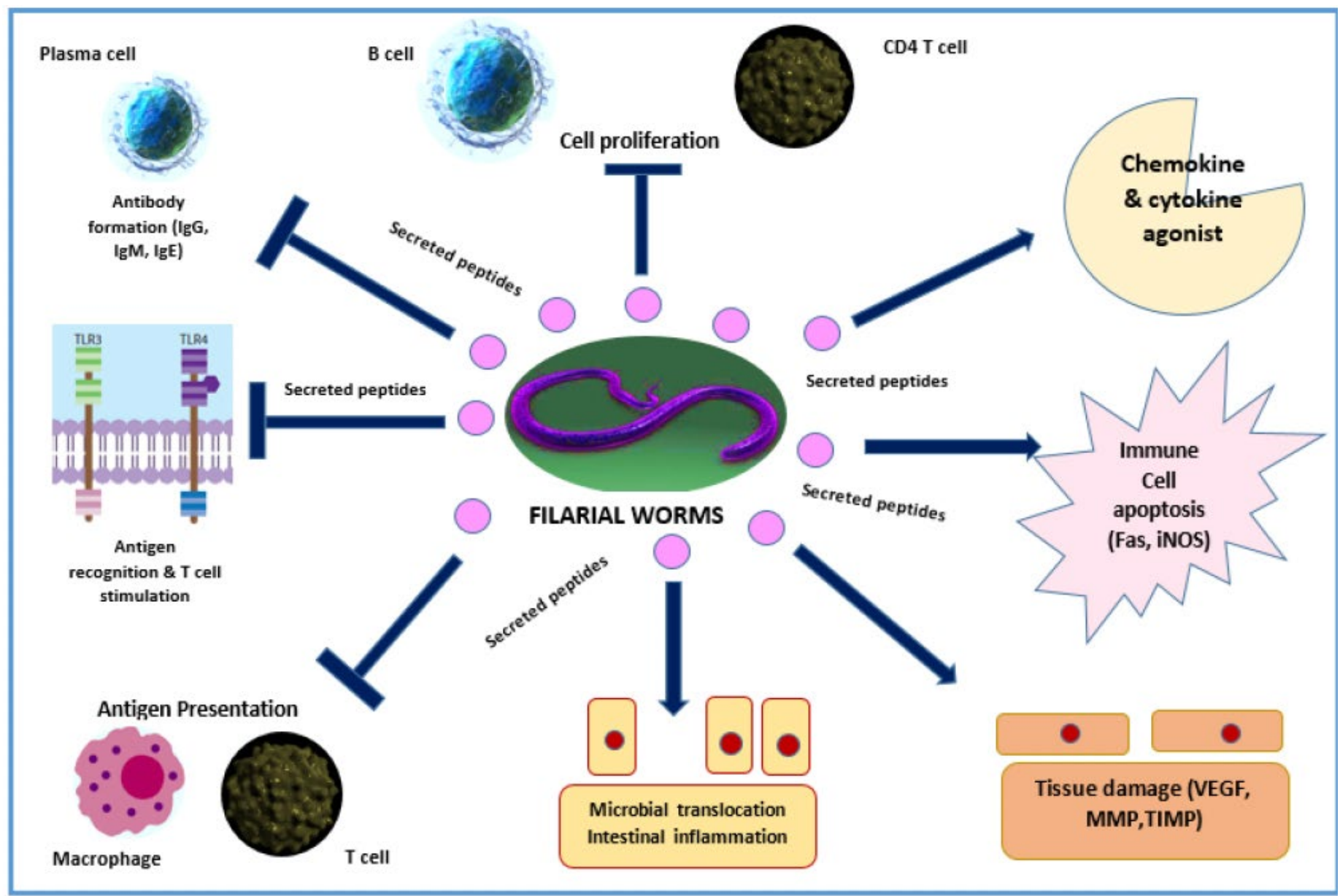

Figure 1: Summary of the immunobiological aspects of the lymphatic filarial worms.

Fas: transmembrane receptor protein; iNOS: inducible nitric oxide synthase; MMP; matrix metalloproteinases; TIMP: tissue inhibitor metalloproteinase; VEGF: vascular endothelial growth factor.

Products secreted by lymphatic filarial worms affect the expression of the Toll-like receptor proteins (TLR 3 and 4 ) in cells such as B cells, T cells, and monocytes, which in turn leads to poor antigen presentation and immune response. ${ }^{26}$ Filarial worms can also stimulate the expression of negative co-stimulatory molecules (receptors) such as cytotoxic T-lymphocyte antigen-4 (CTLA-4) or programmed cell death-1 (PD-1) for T-cell downregulation. ${ }^{27}$

\section{Cellular Effects of the Lymphatic Filarial Worms}

\section{Lymphatic filariasis and T cells}

Transcriptional activators T-bet (master Th1 cell activation factor) and GATA-3 (principal Th2 activation factor) are needed for CD4+ T-cell differentiation. Babu et al. $^{28}$ noted that chronic filarial state affects the activities of these factors, leading to diminished T-cell differentiation and increased expression of factors such as FOXP3, TGF- $\beta$, CTLA-4, PD-1, ICOS (inducible T-cell costimulator), and indoleamine 2,3-dioxygenase with the addition of anergy-inducing factors to human hosts such as cbl-b, c-cbl, Itch, and Nedd4. ${ }^{28}$ An increase also in the frequency of CD8+ $T$ cells was observed by Kroidl et al., ${ }^{29}$ which can be critical, especially during active infection. One potential mechanism is the filarial worm-induced modulation of the expression of chemokine receptor CCR9, which later promotes CD8+ formation. ${ }^{30}$

\section{Lymphatic filariasis and $\mathrm{T}$ regulatory cells}

$T$ regulatory cells, especially with the phenotype (CD25+FOXP3+), are activated by filarial worm infection. ${ }^{23}$ These cells can 
down-regulate other CD4+ subsets by the release of immunoregulatory cytokines such as IL-10 and TGF- $\beta$. This can help the immune evasion of filarial worms by down-regulating effector $T$ cell responses. Another mechanism is by induction of negative co-stimulatory molecules such as CTLA-4 and PD-1 to turn off T-cell activation. ${ }^{27}$

\section{Lymphatic filariasis, macrophages, and dendritic cells}

Macrophage presentation with CD4+ $T$ cells is also down-regulated, especially during the chronic phase. Babu et al. ${ }^{23}$ noted that filarial infections stimulate macrophages to release arginase- 1 to inhibit the release of proliferation cytokines in $T$ cells and induce expression of negative co-stimulatory molecules. The MHC I and // genes are down-regulated, leading to diminished T-cell activation to mount normal anti-filarial immune response.

During an early phase of infection or during tissue damage, filarial worms can trigger an inflammatory response. A $70 \mathrm{kDa}$ microfilarial protein from $W$. bancrofti sheath was isolated by Mukherjee et al..$^{31}$ that can bind to macrophageTLR4 and activates nuclear factor (NF-kB), which then upregulates the release of pro-inflammatory cytokines. Similarly, Mukherjee et al. ${ }^{32}$ noted that microfilarial protein can also activate dendritic cells and drive Th1 responses.

\section{Lymphatic Filariasis and Microbiota}

Increased levels of inflammatory cytokines can stimulate damage to the mucous layer of the intestinal barrier, promote microbial dysbiosis and cause microbial translocation across different sites distal to the intestinal tract, especially in the lymphatics. The presence of microbial products like lipopolysaccharide can trigger activation of host responses such as the complement pathway (alternative pathway) in which systemic effects such as fever and inflammation can add burden to the current state of the disease. Inflammatory compounds seen in chronic lymphatic filariasis include C-reactive protein, TNF-a, IL-2, IL-6, IL-8, endothelin-1, MIP-1a, MIP-1B, MCP-1, TARC, and IP-10. ${ }^{23}$

\section{Lymphatic Filarial Worms and Tissue Damage}

Physical damage due to the migration of adult worms and larvae can induce damage to the connective tissue of the host. The filarial worms along with their secreted products cause imbalance between the matrix metalloproteinases and their inhibitors. This can cause progression into a fibrotic state and matrix remodelling. ${ }^{23}$ Babu et al. ${ }^{23}$ noted that the lymphangiogenic potential (or formation of new lymph vessels within a current lymph tissue) is also brought about by the host secretion of vascular endothelial growth factors $A$ and $C .{ }^{23}$ These growth factors can stimulate an increased vascular diameter, secretion of pro-inflammatory cytokines, and fluids (lymph and plasma), which altogether can result in oedema, lymphatic dysfunction, and could be one of the underlying mechanisms of hydrocele formation in lymphatic filarial worm infection. Filarial worms can also stimulate apoptosis by up-regulation of inducible nitric oxide synthase and Fas for apoptosis of dendritic cells, natural killer cells, and CD4+ T cells in mice models. ${ }^{33}$ This will be another mechanism of immune evasion as these cells play a big role in human host defence against filarial infections.

\section{Anti-inflammatory Potential of the Lymphatic Filarial Worms}

It is known that helminths such as filarial worms can skew Th1 responses into the Th2 phenotype. The former entails activation of cytotoxic $T$ cells and inflammatory cytokines while the latter invokes IL-4 and antibody production. ${ }^{31}$ Hence, Th1 can be essential for the elimination of filarial worms during acute infections or to activate the immune system. Th2 is required to shift from an inflammatory into an anti-inflammatory state and entails the production of protective antibodies.

Filarial worm infected individuals have lowered Th1 and Th17 responses and this could be attributed to expression of negative co-stimulatory molecules such as CTLA-4 and PD-1 receptor. ${ }^{34}$ Metenou et al. ${ }^{36}$ noted that filarial worms can decrease inflammatory genes brought by malarial infection and Babu et al. ${ }^{35}$ noted that exposure of macrophages to filarial worms reduces the important receptors needed for invasion of Mycobacterium tuberculosis. An exciting area worth exploring for immunological 
research and development is to utilise the metabolites of filarial worms, notably antiinflammatory secretory peptides against pathogens such as severe acute respiratory syndrome coronavirus 2 (SARS-CoV-2).

\section{Lymphatic Filarial Worms and Bacterial Endosymbiosis}

Wolbachia spp., an intracellular alphaproteobacteria, are essential for filarial worm fertility, viability, and are commonly found in the female reproductive tract of filarial worms. Phylogenetic analysis noted that Wolbachia spp. are transferred after the divergence of nematodes and arthropods. ${ }^{37}$ Drug regimens for Wolbachia spp. are of long duration and contraindicated for children and pregnant individuals. Microbial and possibly filarial metabolites can potentially be used in alternative treatments for antimicrobialresistant pathogens. Another important note is that the presence of Wolbachia spp. in mosquitos can reduce infections brought by Plasmodium spp., dengue, Chikungunya, and West Nile virus. ${ }^{38}$ This can be due to reducing the mosquito's life span or triggering the mosquito to mount an immune attack against these pathogens.

\section{CO-INFECTIONS: FILARIASIS, MALARIA, AND TUBERCULOSIS}

Tuberculosis is a communicable respiratory disease caused by the aerobic acid-fast bacteria M. tuberculosis. ${ }^{36}$ Bacillus Calmette-Guérin vaccination offers a Th1 response. Exposure to helminths may dampen the response against the vaccine by TGF- $\beta$ production and possibly shifting to Th2 responses. ${ }^{36}$ Patients with onchocerciasis have lowered responses to purified protein derivative. ${ }^{39}$ In a study by Potian et al. ${ }^{40}$ using mouse models infected with a helminth model Nippostrongylus brasiliensis, macrophages were activated by IL-4R and caused resistance to tuberculosis. Latent tuberculosis is exemplified by release of pro-inflammatory cytokines such as IL-1B, IL-6, IL-8, and IL-12; however, with an onset of early to chronic filarial infection, a mixed to down-regulatory cytokine phenotype is noted by Potian et al. ${ }^{40}$

Babu et al..$^{35}$ demonstrated that macrophages and dendritic cells previously infected with filarial worms and exposed to M. tuberculosis showed decreased surface expression of receptors used by the bacterium in invasion.

Malaria is a protozoan infection caused by the genus Plasmodium spp., and co-infections with lymphatic filarial worms have been noted. These two parasites differ in terms of human immune responses. ${ }^{36}$ Plasmodium spp. infections are characterised by production of pro-inflammatory cytokines and Th1 responses while filarial infections are characterised by Th2, IL-10, and T-regulatory cell profiles. Yan et al. ${ }^{41}$ showed that using irradiated $B$. pahangi with Plasmodium berghei infected erythrocytes protected CBA/J mice models against cerebral malaria and caused resistance. This activity could be explained by the IL-10 mechanism and reduced cerebral T cell infiltration. There is also down-regulation of the Th1 response during co-infections, which could possibly be due to IL-12 modulation and a decrease in malarial-induced inflammatory cytokines such as IFN regulatory factor, TNF-a, Th17, IL-12p70, CXCL-10, and IFN-Y. ${ }^{36}$

With regards to Iymphatic filarial worm and malarial parasites on the same vector, the filarial infection in mosquitos can physically damage the midgut tissue and, as a consequence, the ookinete cannot develop. ${ }^{42}$ Filarial worms can also induce immune attack by mosquitoes against invading Plasmodium and leakage of resident bacteria, which produces anti-parasitic metabolites against malaria. Muturi et al. ${ }^{43}$ noted that a filarial worm and a malarial parasite can infect the same human host, as seen in $W$. bancrofti and P. falciparum co-infection.

\section{FUTURE PERSPECTIVES}

Trial vaccines are available for Iymphatic filarial worms. Joseph et al. ${ }^{44}$ determined that multivalent fusion protein vaccine ( $\mathrm{BBmHAT}$ ) with heat shock proteins, larval transcript-2, and tetraspanin conferred $>95 \%$ protection against $B$. malayi infective larvae in mouse and gerbil models. It also enhanced Th2 response against filarial worms. In a related study by Dakshinamoorthy et al., ${ }^{45}$ vaccinated monkeys developed significant titres of antigen-specific IgG antibodies against each of the component antigens rBmHSP12.6, rBmALT-2, and rBmTSPLEL. ${ }^{45}$ Altogether, this is promising for vaccination against Iymphatic filarial worms. The impact of 
lymphatic filarial worms in host microbiota and genetic polymorphisms for vaccine development can also be integrated in the future.

Circulating antigens of $W$. bancrofti are detected by monoclonal antibodies against Og4C3 and AD12 antigens. The determination of the circulating filarial antigens is now considered by the WHO as the 'gold standard'. Both enzyme-linked immunosorbent assay and immunochromatography test formats can detect IgG-4 antibodies against the recombinant BmR1 antigen of B. malayi. Polymerase chain reactionrestriction fragment length polymorphismbased assay using ITS1 rRNA gene as primer can differentiate all the species of human and animal filarial parasites. COX1 gene can also identify between $B$. malayi and B. timori. $7,46,47$

Diethylcarbamazine has been the drug of choice for lymphatic filariasis. ${ }^{48}$ It disrupts the parasitic membrane and is effective for adult worms and microfilaria. Diethylcarbamazine can potentially be used against COVID-19, as it displays antiviral (RNA) activity against murine leukaemia virus. The anti-inflammatory mechanism works by inhibiting cyclooxygenase, promoting antibody production and cytokine release, as well as enhancing Th1 release. ${ }^{49-51}$

Ivermectin is noted for the treatment of lymphatic filariasis, loaiasis, and onchocerciasis. Heidary and Gharebaghi ${ }^{22}$ noted that this drug causes paralysis of the microfilaria and hyperpolarisation of glutamate-sensitive channels. It could potentially be used against COVID-19 as it can inhibit nuclear import of SARS-CoV-2.52 In a randomised, double-blind, placebo-controlled trial by Chaccour et al. ${ }^{53}$ using a single dose of $400 \mathrm{mcg} / \mathrm{kg}$ ivermectin, a reduction of viral load, IgG titres, and anosmia were observed. This highlights the potential positive impact of repurposing anti-filarial drugs against COVID-19.

With the discovery of the bacterial endosymbiont, Wolbachia spp., studies have shown that upon treatment with doxycycline, it effectively decreased and caused the death of adult worms and improved the severity of the lymphoedema. ${ }^{38}$ Doxycycline is also a promising drug against COVID-19 as it can potentially target host proteases (which are needed for preprocessing of SARS-CoV-2) and inhibit matrix metalloproteinases critical for viral fusion. ${ }^{54}$ it also has anti-viral (RNA) and anti-inflammatory effects. ${ }^{55}$ Repurposing of anti-filarial drugs against COVID-19 is a potential area worth exploring.

\section{SUMMARY}

The normal host immune response against lymphatic filarial worms entails CD4+ (Th2) cells, antibodies, complement, phagocytes, and cytokines. Immunological actions of filarial worms entail down-regulation of $B$ and $T$ cells, inhibiting antigen recognition, and stimulation of apoptosis. Secreted peptides of lymphatic filarial worms have potential anti-inflammatory effects. Drug repurposing will also be vital against COVID-19.

\section{References}

1. Center for Disease Control and Prevention (CDC). Parasites Iymphatic filariasis. Epidemiology and risk factors. Available at: https://www. cdc.gov/parasites/lymphaticfilariasis/ epi.html. Last accessed: 3 August 2021

2. Walker PF, Barnett ED (eds.) Immigrant Medicine (2007). $1^{\text {st }}$ edition, Philadelphia: Saunders, Elsevier.

3. Belizario VY Jr, de Leon WU (eds.), Medical Parasitology in the Philippines (2015), $3^{\text {rd }}$ edition, Manila: University of the Philippines Press.

4. Turkington C, Dover JS. The Encyclopedia of Skin and Skin Disorders (2002), $2^{\text {nd }}$ edition, New York: Facts On File.

5. Coutts SP et al. Prevalence and risk factors associated with lymphatic filariasis in American Samoa after mass drug administration. Trop Med Health. 2017;45:22

6. Chesnais $\mathrm{CB}$ et al. Risk factors for lymphatic filariasis in two villages of the Democratic Republic of the Congo. Parasit Vectors. 2019;12(1):162

7. Sastry AS, Bhat S. Essentials of Medical Parasitology (2014), $1^{\text {st }}$ edition, New Delhi: Jaypee Brothers Medical Publishers.

8. Aoki $Y$ et al. Basic studies on filaria and filariasis. Trop Med Health. 2011;39(1 Suppl 2):51-5.

9. Newman TE, Juergens AL. "Filariasis." StatPearls [Internet]. Treasure Island, Florida: StatPearls Publishing. Available at: https://www.ncbi.nlm. nih.gov/books/NBK556012/. Last assessed: 3 August 2021
10. Cross JH. "Filarial Nematodes," Baron S (ed.). Medical Microbiology (1996) $4^{\text {th }}$ edition. Galveston, Texas: University of Texas Medical Branch at Galveston. Available from: https:// www.ncbi.nlm.nih.gov/books/ NBK7844/. Last assessed: 3 August 2021

11. Chandra G. Nature limits filarial transmission. Parasit Vectors. 2008;1(1):13.

12. Li BW et al. Gender-associated genes in filarial nematodes are important for reproduction and potential intervention targets. PLoS Negl Trop Dis. 2011;5(1):e947.

13. Fischer $\mathrm{P}$ et al. Persistence of Brugia malayi DNA in vector and nonvector mosquitoes: implications for xenomonitoring and transmission monitoring of lymphatic filariasis. Am 
J Trop Med Hyg. 2007;76(3):502-7.

14. Partono $\mathrm{F}$ et al. Epidemiological and clinical features of Brugia timori in a newly established village, Karakuak, West Flores, Indonesia. Am J Trop Med Hyg. 1978;27(5):910-5.

15. Joshi, PL. "Elimination of lymphatic filariasis." Letter from Dr. Joshi to National Vector Borne Disease Control Programme in the Government of India. 2006. Available at: https://web.stanford.edu/group/ parasites/ParaSites2006/Lymphatic_ filariasis/References.htm . Last accessed:

16. Chandy A et al. A review of neglected tropical diseases: filariasis. Asian Pac J Trop Med. 2011;4(7):581-6.

17. Babu S, Nutman TB. Immunopathogenesis of lymphatic filarial disease. Semin Immunopathol. 2012;34(6):847-61.

18. Rajan et al. Role of nitric oxide in host defense against an extracellular, metazoan parasite, Brugia malayi. Infect Immun. 1996;64(8)3351-3.

19. Ebbo $M$ et al. Pathologies associated with serum IgG4 elevation. Int J Rheumatol. 2012;2012:602809.

20. Dabir P et al. Immunoprophylactic evaluation of a 37-kDa Brugia malayi recombinant antigen in lymphatic filariasis. Clin Microbiol Infect. 2006;12(4):361-8.

21. Liu LX, Weller PF. Intravascular filarial parasites inhibit platelet aggregation. Role of parasite-derived prostanoids. J Clin Invest. 1992;89(4):1113-20.

22. Senbagavalli $P$ et al. Heightened measures of immune complex and complement function and immune complex-mediated granulocyte activation in human lymphatic filariasis. Am J Trop Med Hyg. 2011;85(1):89-96.

23. Babu S, Nutman TB. Immunology of lymphatic filariasis. Parasite Immunol. 2014;36(8):338-46.

24. Semnani RT et al. Filaria-induced immune evasion: suppression by the infective stage of Brugia malayi at the earliest host-parasite interface. J Immunol. 2004;172(10):6229-38.

25. Desjardins CA et al. Genomics of Loa loa, a Wolbachia-free filarial parasite of humans. Nat Genet. 2013;45(5):495-500.

26. Semnani RT et al. Inhibition of TLR3 and TLR4 function and expression in human dendritic cells by helminth parasites. Blood. 2008;112(4):1290-8.

27. van der Werf $\mathrm{N}$ et al. Th2 cellintrinsic hypo-responsiveness determines susceptibility to helminth infection. PLoS Pathog. 2013;9(3):e1003215.

28. Babu $S$ et al. Diminished expression and function of TL in lymphatic filariasis: a novel mechanism of immune dysregulation. J Immunol. 2005;175(2):1170-6.
29. Kroidl I et al. Wuchereria bancrofti infection is linked to systemic activation of CD4 and CD8 T cells. PLoS Negl Trop Dis. 2019;13(8):e0007623.

30. Johansson-Lindbom B et al. Selective generation of gut tropic $T$ cells in gut-associated lymphoid tissue (GALT): requirement for GALT dendritic cells and adjuvant. J Exp Med. 2003;198(6):963-9.

31. Mukherjee $\mathrm{S}$ et al. A novel ligand of toll-like receptor 4 from the sheath of Wuchereria bancrofti microfilaria induces proinflammatory response in macrophages. J Infect Dis. 2017;215(6):954-65

32. Mukherjee S et al. Wuchereria bancrofti filaria activates human dendritic cells and polarizes $T$ helper 1 and regulatory $T$ cells via toll-like receptor 4. Commun Biol. 2019;2:169.

33. Semnani RT et al. Brugia malayi microfilariae induce cell death in human dendritic cells, inhibit their ability to make IL-12 and IL10 , and reduce their capacity to activate CD4+ T cells. J Immunol. 2003;171(4):1950-60.

34. Babu $\mathrm{S}$ et al. Human type 1 and 17 responses in latent tuberculosis are modulated by coincident filarial infection through cytotoxic $T$ Iymphocyte antigen-4 and programmed death-1. J Infect Dis. 2009;200(2):288-98.

35. Babu $\mathrm{S}$ et al. Attenuation of Toll-like receptor expression and function in latent tuberculosis by coexistent filarial infection with restoration following antifilarial chemotherapy. PLoS Negl Trop Dis. 2009;3(7):e489.

36. Metenou $\mathrm{S}$ et al. Impact of filarial infections on coincident intracellular pathogens: Mycobacterium tuberculosis and Plasmodium falciparum. Curr Opin HIV AIDS. 2012;7(3)231-8

37. Fenn $\mathrm{K}$ et al. Phylogenetic relationships of the Wolbachia of nematodes and arthropods. PLoS Pathog. 2006;2(10):e94.

38. Slatko BE et al. Wolbachia endosymbionts and human disease control. Mol Biochem Parasitol. 2014:195(2):88-95

39. Soboslay PT et al. Ivermectinfacilitated immunity in onchocerciasis. Reversal of lymphocytopenia, cellular anergy and deficient cytokine production after single treatment. Clin Exp Immunol. 1992;89(3):407-13.

40. Potian JA et al. Preexisting helminth infection induces inhibition of innate pulmonary antituberculosis defense by engaging the IL-4 receptor pathway. J Exp Med. 2011;208(9):1863-74

41. Yan $Y$ et al. Down-regulation of murine susceptibility to cerebral malaria by inoculation with third-stage larvae of the filarial nematode Brugia pahangi. Parasitology. 1997;114(Pt 4):333-8.

42. Aliota MT et al. Filarial worms reduce Plasmodium infectivity in mosquitoes. PLoS Negl Trop Dis. 2011;5(2):e963.

43. Muturi EJ et al. Concomitant infections of Plasmodium falciparum and Wuchereria bancrofti on the Kenyan coast. Filaria J. 2006;5:8.

44. Joseph SK, Ramaswamy K. Single multivalent vaccination boosted by trickle larval infection confers protection against experimental lymphatic filariasis. Vaccine. 2013;31(33):3320-6.

45. Dakshinamoorthy $\mathrm{G}$ et al. Evaluation of a multivalent vaccine against ymphatic filariasis in rhesus macaque model. PloS One. 2014;9(11):e112982.

46. Fischer $\mathrm{P}$ et al. Lymphatic filariasis and Brugia timori: prospects for elimination. Trends Parasitol. 2004;20(8):351-5.

47. Fink DL et al. Toward molecular parasitologic diagnosis: enhanced diagnostic sensitivity for filarial infections in mobile populations. J Clin Microbiol. 2011;49(1):42-7.

48. Zulfiqar H, Malik A. Bancroftian Filariasis. 2021. Available at: https:// www.statpearls.com/articlelibrary/ viewarticle/18128/. Last accessed: 6 July 2021

49. Kitchen LW et al. Effect of administration of diethylcarbamazine on murine leukemia virus (Cas- $\mathrm{Br}-\mathrm{M}$ ) infected mice. J Clin Lab Immunol. 1990;33(3):97-105.

50. Medina-De la Garza CE et al. Immunomodulatory activity of diethylcarbamazine on humoral, cellular cytokine response and respiratory burst in BALB/C mice. Immunopharmacol Immunotoxicol. 2012;34(3):477-83.

51. Peixoto CA, Silva BS. Antiinflammatory effects of diethylcarbamazine: a review. Eur J Pharmacol. 2014;734:35-41.

52. Heidary F, Gharebaghi R. Ivermectin: a systematic review from antiviral effects to COVID-19 complementary regimen. J Antibiot (Tokyo). 2020;73(9):593-602.

53. Chaccour $C$ et al. The effect of early treatment with ivermectin on viral load, symptoms and humoral response in patients with non-severe COVID-19: a pilot, double-blind, placebo-controlled, randomized clinical trial. EClinicalMedicine. 2021;32:100720.

54. Phillips JM et al. Neurovirulent murine coronavirus JHM.SD uses cellular zinc metalloproteases for virus entry and cell-cell fusion. J Virol. 2017;91(8):e01564-16.

55. Castro JZ, Fredeking T. Doxycycline modify the cytokine storm in patients with dengue and dengue hemorrhagic fever. Int J Infect Dis. 2010;14(Suppl 1):e44. 